http://jmscr.igmpublication.org/home/ ISSN (e)-2347-176x ISSN (p) 2455-0450 crossref DOI: https://dx.doi.org/10.18535/jmscr/v9i4.12

\title{
Vitamin C supplementation as adjuvant analgesic therapy in chronic backache with radicular symptoms
}

\author{
Authors \\ Neetin Mahajan, Mrugank Narvekar, Lalkar Gadod, Prasanna Kumar G.S., \\ Ishan Gajbe \\ Department of Orthopaedics, Grant Government Medical College, Mumbai, Maharashtra, India \\ *Corresponding Author \\ Dr Mrugank A Narvekar
}

\begin{abstract}
Background: Chronic low back pain (LBP) forms one of the heaviest burdens of disease. Vitamin C has shown analgesic effects in specific clinical conditions, reducing patient suffering and improving the quality of life. The aim of this study was to evaluate the efficacy of vitamin $C$ as an adjuvant analgesic in treatment of chronic low back ache with radicular pain.

Methods: The present study was a prospective study of 100 patients aged 25-65 years with chronic low back ache with radicular pain, conducted in a tertiary care institute from 2019 to 2020. Patients were thoroughly interviewed and the radicular pain was graded according to the Numeric Rating Scale (NRS). 50 patients each were randomized into two groups, those that were given vitamin $c$ supplementation (Group A) and those that weren't (Group B). The patients were then followed up fortnightly for 2 months and the radicular pain was graded at each follow-up.

Results: The mean improvement of the NRS scores in Group A was $51.36 \%$ whereas in Group B was 16.61\%.In Group A, $64 \%$ of patients had good improvement, 14\% moderate improvement while $14 \%$ had mild improvement, and $8 \%$ had no relief. In Group B, $40 \%$ of patients had moderate improvement, $28 \%$ had mild improvement, and $32 \%$ had no relief.

Conclusions: In this comparative study, we suggest that the supplementation of vitamin $c$ to patients experiencing chronic LBP with radicular symptoms acts as an effective adjuvant analgesic giving relief of radicular symptoms with good improvement in the activities of daily living.

Keywords: Vitamin C, Chronic low backache, Radicular pain.
\end{abstract}

\section{Introduction}

Chronic Low back pain is highly prevalent in the general population being one of the major causes of disability as compared to other health conditions. ${ }^{(1,2)}$ Low back pain forms one of the most common forms of chronic pain seen in outpatient departments. Patients often complain of associated radicular pain with the low backache. ${ }^{(3)}$
Radicular pain refers to the pain arising in the lumbar region and radiating in the leg along the course of the affected nerve root. Patients may also have associated symptoms of neurogenic claudication including paraesthesia. The above symptoms impair the patient's quality of life with increased usage of health care resources. 
The treatment guidelines for back pain and radicular pain include a wide variety of options like pharmacological management, physical and rehabilitation measures, and surgical intervention techniques. Patients complaining of associated radicular pain are significantly more likely to take pharmacological treatment than those with axial pain only. Commonly prescribed drugs include NSAIDs, skeletal muscle relaxants, opioid analgesics, benzodiazepines, systemic corticosteroids antidepressants, and anticonvulsants. $^{(3)}$ Though anticonvulsants like gabapentin have been used for the treatment of the associated radicular pain with the low back pain, studies show that there is no treatment benefit in regards to pain and disability with these drugs but are accompanied by an increased risk of adverse events. $^{(3)}$

Reactive oxygen species (ROS), such as superoxide, hydrogen peroxide, and hydroxyl radicals, arising from the metabolism of molecular oxygen $^{(4)}$, have been shown to sensitize the pain pathways during persistent pain. Elevated production of ROS was demonstrated in the spinal cord and peripheral tissues after noxious hind paw stimulation or nerve injury (4). The administration of free radical scavengers has been shown to mitigate inflammatory and neuropathic pain as evident in animal models ${ }^{(4,5)}$, suggesting that ROS are involved in nociceptive processing.

Vitamin C (L-ascorbic acid) is a water-soluble acid capable of scavenging ROS at physiological $\mathrm{pH}^{(6)}$. Suboptimal vitamin $\mathrm{C}$ levels have been associated with spinal and musculoskeletal pain. (7) Further, there have been published reports about the analgesic effect of Vit $\mathrm{C}$ in both animals and humans experiencing neurogenic pain. $(4,8,9)$ Clinical studies indicate a reduction in the symptoms of Chronic regional pain syndrome, acute herpetic neuralgia, postherpetic neuralgia, and cancer-related pain with the administration of high-dose Vitamin C. The oxidative properties of vitamin $\mathrm{c}$ have been found to modify neuralgic pain. Vitamin $C$ can thus be regarded as a safe and effective adjuvant in the treatment of acute and chronic pain in a certain group of patients.

Back pain forms one of the heaviest burdens of disease. Despite much research, this condition remains poorly understood, with a dearth of effective treatments. The aim of this study was to evaluate the effect of vitamin c administration in patients complaining of persistent low back pain with associated radicular pain not relieved by other conservative measures (NSAIDs and physiotherapy) in suppressing the pain.

\section{Materials and Methods}

The present study was a prospective study, conducted in a tertiary care institute from 2018 to 2019 with prior approval from the institutional ethics committee. The study population comprised of the patients presenting to the institute with chronic low backache with associated radicular pain persistent despite other conservative treatments (NSAIDs and physiotherapy) who satisfied the selection criteria for the study.

A total of 100 patients presenting to our tertiary care institute with chronic low backache with associated radicular pain were selected for the study. Written valid informed consent was obtained from the patients at the first presentation. The 100 patients were serially numbered from 1 to 100 as per chronology of presentation and 50 patients each were randomized into two groups, those that were given vitamin c supplementation (Group A) and those that weren't given vitamin c supplementation (Group B). Randomization was done using a Random number table.

\section{Inclusion Criteria}

Patients of either sex in the range of 25-65 years with normal neurology without any claudication symptoms were included in the study. The patients had minimal disc space reduction on $\mathrm{x}$ rays with MRI showing annular tear and bulges in the dorsolumbar region with minimal degenerative changes in the lumbar spine. Patients were not receiving pain modulation therapy like Transcutaneous Electrical Nerve Stimulation or acupuncture therapy. 


\section{Exclusion Criteria}

Patients with any pathological diseases of the spine including DISH, spondylolisthesis, spondylolysis, inflammatory conditions, benign or secondary tumors, and infective pathology of the spine. Patients with MRI showing severe canal stenosis, disc prolapse. Patients having undergone spinal surgeries previously were excluded from the study.

Patients meeting the inclusion and exclusion criteria were interviewed and the radicular pain was graded according to the Numeric Rating Scale (NRS). Pain intensity over the last 24 hours was measured on an 11-point pain scale, anchored at "no pain" and "worst pain I have ever experienced." Patients in Group A were then started on supplementation of vitamin c 1000$1500 \mathrm{mg} /$ day dosing orally. The patients were then followed up on a fortnightly basis for 2 months and the radicular pain was graded at each follow-up. Reduction of radicular pain by more than $(20 \%)$ from the start of vitamin c supplementation was taken to be significant.

\section{Results}

The study was conducted in a tertiary care hospital after obtaining permission from the Institutional Ethics Committee. A total of 100 cases of chronic low backache with associated radicular pain were included in the present study. The patients were divided into 2 groups, i.e. Group A - patients administered vitamin C, and Group B - patients not supplemented with vitamin $\mathrm{C}$.

The average age of the patients in Group A was 46.22 years and of the patients in Group B was 47.26 years in the range of $25-65$ years. The difference in the mean ages of the two groups was not statistically significant ( $p>0.05$ ). In our study, the majority $(60 \%)$ of the patients were females. There were 18 males and 32 females in Group A and 22 males and 28 females in Group B. In the present study, maximum patients $70(70 \%)$ were from the lower class, 20(20\%) from the middle class, and $10(10 \%)$ were from high class society (socio economic status).
In Group A, 33(66\%) of patients had moderate pain score, $12(24 \%)$ had severe pain score and $5(10 \%)$ had mild pain and in Group B, 34(68\%) of patients had moderate pain score, 10(20\%) had severe pain score and $6(12 \%)$ had mild pain score as per NRS score.

In Group A, 44(88\%) of patients had limitation of daily activities due to LBA, and the daily activities of $6(12 \%)$ patients were not affected. In Group B, 40(80\%) of patients had a limitation of daily activities due to LBA, and the daily activities of 10(20\%) patients were not affected.

Table 1 : Mean NRS scores at each follow up

\begin{tabular}{|l|c|c|}
\hline \multirow{2}{*}{ Followup (weeks) } & \multicolumn{2}{|c|}{ Mean NRS scores } \\
\cline { 2 - 3 } & Group A & Group B \\
\hline 0 & 5.66 & 5.54 \\
\hline 2 & 5 & 5.7 \\
\hline 4 & 3.86 & 5.38 \\
\hline 8 & 2.66 & 4.6 \\
\hline
\end{tabular}

The difference in the mean NRS scores at 0 weeks was not statistically significant $(\mathrm{p}>0.05)$. The differences between the mean NRS scores at 2, 4, and 8 week follow-ups were statistically significant $(\mathrm{p}<0.05)$

The mean improvement of the NRS scores in Group A was $51.36 \%$ whereas in Group B was $16.61 \%$. In Group A, 64\% of patients had good improvement after treatment protocol followed by us. $14 \%$ of patients had moderate improvement while $14 \%$ had mild improvement. Only $8 \%$ of patients had no relief in their pain. In Group B, $40 \%$ of patients had moderate improvement while $28 \%$ had mild improvement. $32 \%$ of patients had no relief in their pain.

Table 2: Mean outcome according to the age group

\begin{tabular}{|l|c|c|}
\hline & \% improvement(mean) & \\
\hline Age & Group A & Group B \\
\hline $30-40$ & 56.1 & 17 \\
\hline $40-50$ & 61.17 & 20.33 \\
\hline $50-60$ & 36.28 & 11.91 \\
\hline $60-70$ & 31.66 & 9 \\
\hline
\end{tabular}

The reduction in radicular pain was higher in males as compared to females. In Group A, the mean reduction in radicular pain was $62.38 \%$ in males as compared to $44.62 \%$ in females. In 
Group B, the mean reduction in radicular pain was $18.86 \%$ in males as compared to $14.78 \%$ in females.

\section{Discussion}

Low back pain (LBP) forms one of the heaviest burdens of disease. It is one of the most common forms of disability impairing patients' quality of life increasing the total expenditure on pain management per annum.

Suboptimal Vitamin C levels have been associated with spinal pain, primarily neck and lower back, musculoskeletal pain, and arthritis/rheumatism. ${ }^{(1)}$ Clinical studies investigating the effect of vitamin c supplementation in wrist and ankle surgery patients have shown decreased incidence of CRPS with daily administration of vitamin $\mathrm{c}$ with doses $\geq 0.5 \mathrm{~g} /$ day being the most efficacious. ${ }^{(10)}$

Vitamin $\mathrm{C}$ is a potent antioxidant capable of scavenging ROS involved in nociceptive processing. ${ }^{(11)}$ It also exhibits anti-inflammatory properties through unknown mechanisms. It is involved in neuromodulation, as a cofactor in the synthesis of catecholamine neurotransmitters. ${ }^{(12)}$ Vitamin $\mathrm{C}$ is responsible for the recycling of the cofactor tetrahydrobiopterin required in the synthesis of dopamine and serotonin. ${ }^{(13)}$ It also acts as a cofactor in the conversion of dopamine to norepinephrine. The inhibition of serotonin and norepinephrine reuptake is efficacious in the control of pain. ${ }^{(14)}$ Studies also have proven a decrease in opioid dependence in patients receiving vitamin $\mathrm{C}$ supplementation. ${ }^{(15)}$

Oral administrations of vitamin c tablets make it a simple and easy method, used in outpatient clinics. There have been no serious complications reported with vitamin $\mathrm{c}$ administration. No loss to follow-up seen. This therapy helped to improve trust in treating orthopaedic surgeons and improved doctor-patient relationship and bonding. The need for surgical interventions can be easily delayed reducing morbidity associated with surgical interventions. It increases the effectiveness of conservative therapy used for LPB associated radicular pain, commonly used for suspected nerve root inflammation in early degenerative disc disease. Overall, vitamin $\mathrm{C}$ is an effective drug in the armamentarium to control LBP and the associated radicular symptoms.

In the present study, the mean age of the patients in Group A was 46.22 years with the youngest being 34 years and the oldest being 62 years. The majority of the patients, 23 , were in the age group of 40 to 50 years. The mean age of the patients in Group B was 47.26 years with the youngest being 36 years and the oldest being 63 years. The majority of the patients, 24 , were in the age group of 40 to 50 years. The study by Ghai et.al. ${ }^{(16)}$ showed the mean age of 43.8 years in patients with LBP. In a similar study by Goon et. al. ${ }^{(17)}$ $44 \%$ of the population with LBP was above 40 years.

In the present study, the majority of the patients were female in the total study population comprising $60 \%$ of the total population studied and the rest were males (40\%). Group A had 64\% females and $36 \%$ males while in Group B there were $56 \%$ females and $44 \%$ males. In the present study female patients were more, similar to studies by Mohapatra et. al. (18), Bihari et. al. ${ }^{(19)}$, and Banarjee et. al. ${ }^{(20)}$

In the present study, maximum patients $70(70 \%)$ were from the lower-income class, 20(20\%) from the middle-income class, and 10(10\%) were from the upper-income class group. The study by Bandyopadhyay et.al. ${ }^{(21)}$ concluded that workers from the lower-income group had more musculoskeletal problems than workers from higher-income groups $(8.1 \%)$.

The majority $86(86 \%)$ of patients had difficulty in performing daily activities. The study by Sharma et.al. had concluded that $26 \%$ of their subjects had to change their profession due to LBP. ${ }^{(22)}$

The outcome at the end of 8 weeks showed that in Group A $64 \%$ of patients had good improvement after treatment, $14 \%$ patients had moderate improvement while $14 \%$ had mild improvement. Only $8 \%$ of patients had no relief in their pain. In Group B, $40 \%$ of patients had moderate 
improvement while $28 \%$ had mild improvement. $32 \%$ of patients had no relief in their pain.

The maximum improvement was seen in the age group of 40-50 years with a mean reduction in pain in Group A being $61.17 \%$ and Group B $20.33 \%$. The minimum improvement was seen in the elderly age group patients who could be due to other nutritional deficiencies. Male patients showed better improvement in the NRS scores with greater mean improvement as compared to female patients.

At the time of the start of the study, 44 patients $(88 \%)$ in Group A had limitation of activities of daily living, and 6 patients $(12 \%)$ had no limitation of activities of daily living. After completion of the study out of the 44 patients, 41 patients $(93 \%)$ reported good improvement in activities of daily living, and 3 patients (7\%) reported no improvement in their activities of daily living.

Thus, in this comparative study, we suggest that the supplementation of vitamin $\mathrm{c}$ to patients experiencing chronic LBP with radicular symptoms acts as an effective adjuvant analgesic giving relief of radicular symptoms with good improvement in the activities of daily living.

\section{Conclusion}

Vitamin $\mathrm{C}$ has shown analgesic effects in specific clinical conditions, reducing patient suffering and improving the quality of life. Chronic pain is a burden on health care expenditure. It requires treatment, at the same time complicates treatment of other conditions and decreases productivity. In this clinical outcome-based study, we suggest that vitamin $\mathrm{C}$ acts as a cost-effective, safe, and efficacious adjuvant in the treatment of chronic LBP with radicular symptoms.

Future high-quality studies are required to confirm these findings. Future studies should endeavor to ascertain the following aspects: measurement of vitamin $\mathrm{C}$ concentrations at baseline and following intervention to determine if specific patient groups respond, determination of the optimal route of administration (i.e. enteral or parenteral), as the present study is carried out for oral administration of vitamin c.

Source of Support: This research did not receive any specific grant from funding agencies in the public, commercial, or not-for-profit sectors.

Conflict of Interest: The authors have no conflicts of interest to declare.

\section{References}

1. Dionne CE, Laurin $\mathrm{D}$, Desrosiers $\mathrm{T}$, Abdous B, Le Sage N, Frenette J, Mondor $\mathrm{M}$, Pelletier S. Serum vitamin $\mathrm{C}$ and spinal pain: a nationwide study. Pain. 2016 Nov 1;157(11):2527-35.

2. Enke O, New HA, New $\mathrm{CH}$, Mathieson $\mathrm{S}$, McLachlan AJ, Latimer J, Maher CG, Lin CW. Anticonvulsants in the treatment of low back pain and lumbar radicular pain: a systematic review and meta-analysis. Cmaj. 2018 Jul 3;190(26):E786-93.

3. Manchikanti L, Hirsch JA. Clinical management of radicular pain. Expert review of neurotherapeutics. 2015 Jun 3;15(6):681-93.

4. Lu R, Kallenborn-Gerhardt W, Geisslinger $\mathrm{G}$, Schmidtko A. Additive antinociceptive effects of a combination of vitamin $\mathrm{C}$ and vitamin $\mathrm{E}$ after peripheral nerve injury. Plos one. 2011 Dec 14;6(12):e29240.

5. Kim HK, Park SK, Zhou JL, Taglialatela G, Chung K, Coggeshall RE, Chung JM. Reactive oxygen species (ROS) play an important role in a rat model of neuropathic pain. Pain. 2004 Sep 1;111(12):116-24.

6. Kondo Y, Sasaki T, Sato Y, Amano A, Aizawa S, Iwama M, Handa S, Shimada N, Fukuda M, Akita M, Lee J. Vitamin C depletion increases superoxide generation in brains of SMP30/GNL knockout mice. Biochemical and biophysical research communications. 2008 Dec 5;377(1):2916. 
7. Carr AC, McCall C. The role of vitamin $\mathrm{C}$ in the treatment of pain: new insights. Journal of translational medicine. 2017 Dec;15(1):1-4.

8. Li R, Shen L, Yu X, Ma C, Huang Y. Vitamin $\mathrm{C}$ enhances the analgesic effect of gabapentin on rats with neuropathic pain. Life sciences. 2016 Jul 15;157:25-31.

9. Chen JY, Chang CY, Feng PH, Chu CC, So EC, Hu ML. Plasma vitamin $\mathrm{C}$ is lower in postherpetic neuralgia patients and administration of vitamin $\mathrm{C}$ reduces spontaneous pain but not brush-evoked pain. The Clinical journal of pain. 2009 Sep 1;25(7):562-9.

10. Zollinger PE, Tuinebreijer WE, Breederveld RS, Kreis RW. Can vitamin C prevent complex regional pain syndrome in patients with wrist fractures?: A randomized, controlled, multicenter doseresponse study. JBJS. 2007 Jul 1;89(7):1424-31.

11. Buettner GR. The pecking order of free radicals and antioxidants: lipid peroxidation, $\alpha$-tocopherol, and ascorbate. Archives of biochemistry and biophysics. 1993 Feb 1;300(2):535-43.

12. Du J, Cullen JJ, Buettner GR. Ascorbic acid: chemistry, biology and the treatment of cancer. Biochimica et Biophysica Acta (BBA)-Reviews on Cancer. 2012 Dec 1;1826(2):443-57.

13. May JM, Qu ZC, Meredith ME. Mechanisms of ascorbic acid stimulation of norepinephrine synthesis in neuronal cells. Biochemical and biophysical research communications. 2012 Sep 14;426(1):148-52.

14. Mochizucki D. Serotonin and noradrenaline reuptake inhibitors in animal models of pain. Human Psychopharmacology: Clinical and Experimental. 2004 Oct;19(S1):S15-9.
15. Buga S, Sarria JE. The management of pain in metastatic bone disease. Cancer Control. 2012 Apr;19(2):154-66.

16. Babita Ghai MD, Dipika Bansal MD, Raju Kanukula M. High prevalence of hypovitaminosis D in Indian chronic low back patients. Pain Physician. 2015 Sep;18(E853):E853-62.

17. Goon M, Ghoshal S, Chandrasekaran B, Sharma BC. Prevalence of low back pain in long distance truck drivers of mountainous terrain. na; 2010 Jan 1.

18. Mohapatra A, Handoo SK, Gambhir IS, Mohapatra SC, Mohapatra SC. A study of non-communicable morbidity pattern in geriatric patients attending a referral railway hospital in allahabad, uttar pradesh. Nat J Commun Med. 2011;2(2):191-5.

19. Bihari V, Kesavachandran C, Pangtey BS, Srivastava AK, Mathur N. Musculoskeletal pain and its associated risk factors in residents of National Capital Region. Indian journal of occupational and environmental medicine. 2011 May; 15(2):59.

20. Banerjee A, Jadhav SL, Bhawalkar JS. Limitations of activities in patients with musculoskeletal disorders. Annals of medical and health sciences research. 2012;2(1):5-9.

21. Bandyopadhyay A, Dev S, Gangopadhyay S. A study on the prevalence of musculoskeletal disorders among the coalminers of Eastern Coalfields of India. International Journal of Occupational Safety and Health. 2012 Jul 31;2(2):34-7.

22. Sharma SC, Singh R, Sharma AK, Mittal $\mathrm{R}$. Incidence of low back pain in workage adults in rural North India. Indian journal of medical sciences. 2003 Apr 1;57 (4): 145 . 